PANORAMA

ISSN: 1909-7433

ISSN: 2145-308X

ednorman@poligran.edu.co

Politécnico Grancolombiano

Colombia

\title{
MENSAJES DE AUTORREGULACIÓN DEL APRENDIZAJE EN ESTUDIANTES DEL GRADO NOVENO DEL COLEGIO CUNDINAMARCA IED EN BOGOTÁ
}

\author{
Tiusaba-Rivas, Alejandro \\ MENSAJES DE AUTORREGULACIÓN DEL APRENDIZAJE EN ESTUDIANTES DEL GRADO NOVENO DEL \\ COLEGIO CUNDINAMARCA IED EN BOGOTÁ \\ PANORAMA, vol. 15, núm. 29, 2021 \\ Politécnico Grancolombiano, Colombia \\ Disponible en: https://www.redalyc.org/articulo.oa?id=343967896007 \\ DOI: https://doi.org/10.15765/pnrm.v15i29.1724
}

\section{(c) (1)(2)}

Esta obra está bajo una Licencia Creative Commons Atribución-NoComercial-Compartirlgual 4.0 Internacional. 


\section{MENSAJES DE AUTORREGULACIÓN DEL APRENDIZAJE EN ESTUDIANTES DEL GRADO NOVENO DEL COLEGIO CUNDINAMARCA IED EN BOGOTÁ}

Learning self-regulation messages in ninth grade students of colegio Cundinamarca IED in Bogotá

Mensagens de autorregulação da aprendizagem em estudantes do $9^{\circ}$ ano do colégio Cundinamarca IED em Bogotá

PANORAMA, vol. 15, núm. 29, 2021

Politécnico Grancolombiano, Colombia

Recepción: 17 Febrero 2021

Aprobación: 13 Mayo 2021

DOI: https://doi.org/10.15765/ pnrm.v15i29.1724

Redalyc: https://www.redalyc.org/ articulo.oa?id=343967896007
Alejandro Tiusaba-Rivas

Universidad Distrital Francisco José de Caldas, Colombia

https://orcid.org/0000-0002-3596-2563
Resumen: El propósito de este estudio fue analizar las cualidades psicométricas del Cuestionario de Mensajes Autorregulatorios (CMA), en 40 estudiantes del grado noveno del Colegio Cundinamarca IED, en la ciudad de Bogotá. Se adoptó por un diseño no experimental cuantitativo que pretendió observar el fenómeno de la autorregulación del aprendizaje en su contexto natural. Los principales hallazgos revelaron la existencia de una correlación significativa entre el desempeño y el control de las emociones. Esto permitió reconocer que los estudiantes con una baja autorregulación emocional están asociados a altos indicadores de fracaso en tareas y actividades, y que estas suelen generar efectos negativos en las emociones de los individuos, que luego desembocan en una sistemática evitación.

Palabras clave: Autorregulación del aprendizaje, educación, jóvenes, auto-mensajes.

Abstract: The purpose of this study was to analyze the psychometric qualities of the Self-Regulatory Messages Questionnaire (CMA) in 40 ninth-grade students from Colegio Cundinamarca IED in the city of Bogotá. It was adopted by a quantitative non-experimental design that sought to observe the phenomenon of self-regulation of learning in its natural context. The main findings revealed the existence of a significant correlation between performance and control of emotions. This allowed to recognize that students with low emotional self-regulation are associated with high indicators of failure in tasks and activities, and that these tend to generate negative effects on the emotions of individuals, which later lead to systematic avoidance.

Keywords: Self-regulation of learning, education, youngs, self-messages.

Resumo: O objetivo deste estudo foi analisar as qualidades psicométricas do Questionário de Mensagens Auto-regulatórias (CMA) em 40 estudantes do $9^{\circ}$ grau do Colégio Cundinamarca IED na cidade de Bogotá. Foi adotado por um projeto não experimental quantitativo que pretendeu observar o fenômeno da auto-regulação da aprendizagem em seu contexto natural. As principais descobertas revelaram a existência de uma correlação significativa entre o desempenho e o controle das emoções. Isso permitiu reconhecer que alunos com baixa autorregulação emocional estão associados a altos indicadores de fracasso em tarefas e atividades, e que estes tendem a gerar efeitos negativos nas emoções dos indivíduos, que posteriormente levam à evitação sistemática.. 
Palavras-chave: Auto-regulação da aprendizagem, educação, jovens, auto-mensagens.

\section{INTRODUCCIÓN}

El desarrollo de procesos de autorregulación del aprendizaje se ha constituido en uno de los principales desafíos y objetivos de la educación contemporánea. Actualmente, un gran número de instituciones educativas continuamente fomentan y promueven formas específicas de control y dirección de los propios procesos cognitivos y motivaciones a lo largo de las trayectorias educativas de los estudiantes (Ader, 2019). Esto ha ocasionado que en las últimas décadas asciendan aceleradamente estudios investigativos sobre las formas en que los docentes implementan nuevos enfoques y métodos en el aula, y los impactos que estas transformaciones pedagógicas han podido provocar en los procesos de intervención activa que los estudiantes realizan para alcanzar sus objetivos de aprendizaje y el éxito académico (Boekaerts, Pintrich \& Zeidner, 2000; Mega, Ronconi \& de Beni, 2014; Spruce \& Bol, 2015).

Discusiones actuales sobre el reciente interés académico como el de Rosário et al. (2014) por el estudio de la autorregulación del aprendizaje coinciden en plantear que el progresivo reconocimiento social y educativo de los sujetos, como agentes o participantes activos dentro de los procesos cognitivos, motivacionales y conductuales del proceso de aprendizaje ha posibilitado la emergencia paulatina de estudios sobre la autorregulación (Pintrich, 2000; Ruban \& Reis, 2006; Zimmerman, 2008; Muñoz, 2020). Hoy en día se puede afirmar que mediante la consideración del rol dinámico del sujeto dentro del proceso de enseñanza-aprendizaje se favoreció la adopción de posturas teóricas afines al constructivismo, que situaron a los estudiantes como aprendices "autorregulados", en la medida en que comenzaban a ser aceptados como participantes metacognitiva, motivacional y conductualmente activos de su propio aprendizaje (De Corte, Verchaffel \& Eynde, 2011; Zimmerman, 2001; González, 2020).

Con los cambios clave en el rol del sujeto de los últimos años se ha elevado la necesidad de centrar los objetivos educativos en ayudar a los estudiantes a desarrollarse como aprendices autónomos y autorregulados (Föster, 2018; Panadero, 2011). Esto supone para los docentes educar a los estudiantes en cómo autorregular el propio aprendizaje para potenciar habilidades dentro del entorno académico que favorezcan el logro de objetivos (Aguilar, 2020). En otras palabras, implica contribuir en la constitución de sujetos-agentes educativos que sepan "qué, cuándo, cómo, por qué y para qué del aprendizaje" (Panadero, 2011, p.3). Esto, en definitiva, se traduce en conseguir que dentro de la extensa trayectoria escolar el sujeto "aprenda" a ser capaz de autorregular su aprendizaje (Cabero-Almenara, 2020).

Siguiendo a Panadero (2011), la autorregulación del aprendizaje es un proceso complejo que ha sido definido como el control que los sujetos realizan "sobre sus pensamientos, acciones, emociones y motivaciones a través de estrategias personales para alcanzar los objetivos que han establecido" (p.11). Lo anterior contempla, por un lado, el control de los 
pensamientos y de las acciones, es decir, el componente cognitivo, que con frecuencia es llamado metacognición, y el componente conductual que se realiza para alcanzar objetivos. Por otro lado, comprende el control de las emociones, pues recientemente se ha reconocido su centralidad para el aprendizaje. Finalmente, incluye de acuerdo con Panadero (2011), el control de la motivación que radica en "automotivarse para una tarea y en mantener, durante la ejecución, la concentración el interés a través de estrategias" (p.11), con el propósito de alcanzar los objetivos trazados al inicio.

Con lo planteado hasta el momento, el presente estudio intentó comprender la autorregulación del aprendizaje por medio de los mensajes que los estudiantes se dirigen a sí mismos durante el tiempo que realizan una tarea (Panadero, 2011; Wolters, 2003). Se trata, pues, de indagar los pensamientos que pasan por la cabeza de un estudiante que le permiten sostener la concentración y el interés durante el desarrollo de una actividad. Por consiguiente, se puede plantear que algunos de los pensamientos que circulan en la cabeza de un estudiante cuando realiza una tarea suelen expresarse como auto-mensajes que intervienen y controlan estratégicamente la conducta y la motivación hacia el logro del aprendizaje (Corno, 2008; Schunk, 2001). En muchos casos, estos automensajes exteriorizan y refuerzan las expectativas de éxito o fracaso de los estudiantes ante el aprendizaje (Schunk, 2012; Wolters, 2003).

La exploración y el análisis de los auto-mensajes es crucial para poder controlar los pensamientos y su injerencia con el aprendizaje (Dignath \& Büttner, 2008; Kuhl, 2000); En especial, por la función que cumplen a la hora de autorregular conductas, supervisar metas y reforzar todo tipo de emociones positivas o negativas que pueden favorecer el logro de objetivos (Ruipérez-Valiente, 2020). En ese sentido, el objetivo del estudio consistió en analizar las cualidades psicométricas del Cuestionario de Mensajes Autorregulatorios (CMA), utilizado en una muestra de estudiantes del grado noveno del Colegio Cundinamarca IED, con el fin de evaluar opiniones y actitudes que surgen a manera de auto-mensajes en la mente de los estudiantes mientras desarrollan algún tipo de tarea o actividad.

\section{MÉTODO}

El estudio adoptó un diseño no experimental cuantitativo. Este podría definirse como un acercamiento investigativo que se realiza sin manipular deliberadamente variables. Es decir, se trata de un ejercicio en el cual no se modifican en forma intencional las variables independientes para ver su efecto sobre otras (Hernández, Fernández \& Baptista, 2014). Lo que se pretende con este diseño es observar un fenómeno tal como se da en su contexto natural para analizarlo. 


\section{Participantes}

El estudio se llevó a cabo en una muestra de estudiantes $(n=40)$, del grupo 901 de la jornada de la mañana (J.M.) del Colegio Cundinamarca IED, ubicado en la localidad 19 de Ciudad Bolívar en Bogotá. Todos los participantes pertenecían a familias de nivel socioeconómico medio-bajo. La recolección de los datos se realizó durante la última semana del mes de octubre del año 2020.

La muestra estuvo conformada por los casos disponibles a los que a la fecha se tenía acceso como docente en la institución (Battaglia, 2008). Esta conveniencia en la muestra se debió a que el tiempo y los medios de recolección de los datos fueron limitados por la cercanía de las fechas de finalización del periodo académico de la institución. A todos los participantes se les explicó cuál era el objetivo de la aplicación del cuestionario y se les animó a participar de forma voluntaria. No recibieron ningún tipo de incentivo académico por su participación y tuvieron tiempo y espacio extracurricular para dar respuesta al instrumento. Los participantes solo podían responder una vez a los 20 ítems del cuestionario.

El rango de edad de la muestra iba desde los 13 hasta los 17 años $(M=14,95 ; S D=1,02347)$. La distribución de la muestra se indica en la tabla 1.

\begin{tabular}{|c|c|c|}
\hline Grupo & \multicolumn{2}{|c|}{ Participantes } \\
\hline \multirow{4}{*}{ 901J.M. } & Género & Frecuencia \\
\cline { 2 - 3 } & Hombres & 19 \\
\cline { 2 - 3 } & Mujeres & 21 \\
\cline { 2 - 3 } & Total & 40 \\
\cline { 2 - 3 } & Edad & Frecuencia \\
\cline { 2 - 3 } & 13 & 1 \\
\cline { 2 - 3 } & 14 & 15 \\
\cline { 2 - 3 } & 15 & 13 \\
\cline { 2 - 3 } & 16 & 7 \\
\cline { 2 - 3 } & 17 & 4 \\
\cline { 2 - 3 } & Total & 40 \\
\hline
\end{tabular}

Tabla 1.

Distribución de la muestra

\section{Instrumento}

El instrumento empleado fue el Cuestionario de Mensajes Autorregulatorios (CMA) o Self-Regulatory Messages Questionnaire (SRMQ), desarrollado por Alonso-Tapia y Panadero (2010). El cuestionario contó con 20 ítems de 34 (originalmente planteados en su versión en inglés bajo las siglas SRMQ), que evaluaba los probables automensajes que los estudiantes se dirigen a sí mismos durante el tiempo 
que intentan alcanzar metas de aprendizaje. La medida de coherencia o consistencia interna del Cuestionario de Mensajes Autorregulatorios (CMA) fue igual a $\alpha 0,7931$.

El instrumento agrupó cinco factores de la autorregulación del aprendizaje: emociones negativas ( 4 ítems) $\alpha$ 0,718, motivación ( 5 ítems) $\alpha$ 0,761, evitación (2 ítems) $\alpha$ 0,361, desempeño (6 ítems) $\alpha$ 0,644 y proceso (2 ítems) $\propto$ 0,641, y se debía responder en una escala Likert de cinco puntos. El ítem 11 del instrumento no fue evaluado debido a que aún se encuentra en validación para Alonso-Tapia y Panadero (2010).

\section{Análisis de datos}

La recopilación de datos se hizo por medio de un formulario de Google que estuvo disponible durante una semana en línea. Se extrajo cada dato a una hoja de cálculo de Excel que posteriormente fue importada al software SPSS que ofrece IBM para el análisis estadístico. En el estudio de la base de datos se obtuvieron indicadores estadísticos descriptivos y se calcularon coeficientes de correlación para determinar la asociación entre los cinco factores de la autorregulación del aprendizaje (emociones negativas, motivación, evitación, desempeño y proceso).

\section{RESULTADOS}

El instrumento planteado por Alonso-Tapia y Panadero (2010) se aplicó a una muestra de 40 participantes. Este presentó una media de 2,753 con valores que oscilaron entre 1,800 como mínimo y 3,473 como máximo. El rango de las varianzas del elemento y de las covarianzas entre elementos fue de 0,83 y 1,459 respectivamente. Finalmente, el rango de las correlaciones entre elementos (ítems o reactivos) del instrumento de indagación estuvo en 1,064 con una varianza total igual a 0,057.

\begin{tabular}{|l|c|c|c|c|c|c|c|}
\hline & Media & Mínimo & Máximo & Rango & $\begin{array}{c}\text { Máximo / } \\
\text { Mínimo }\end{array}$ & Varianza & $\begin{array}{c}\text { N de } \\
\text { elementos }\end{array}$ \\
\hline $\begin{array}{l}\text { Medias de } \\
\text { elemento }\end{array}$ & 2,753 & 1,800 & 3,475 & 1,675 & 1,931 &, 244 & 20 \\
\hline $\begin{array}{l}\text { Varianzas de } \\
\text { elemento }\end{array}$ & 1,341 & 1,015 & 1,846 &, 831 & 1,818 &, 051 & 20 \\
\hline $\begin{array}{l}\text { Covariables entre } \\
\text { elementos }\end{array}$ &, 216 &,- 487 &, 972 & 1,459 & $-1,995$ &, 101 & 20 \\
\hline $\begin{array}{l}\text { Correlaciones entre } \\
\text { elementos }\end{array}$ &, 160 &,- 384 &, 679 & 1,064 & $-1,767$ &, 057 & 20 \\
\hline
\end{tabular}

Tabla 2.

Estadísticas resumen de los elementos (ítems o reactivos del instrumento)

Las estadísticas totales de los ítems o elementos del instrumento revelan que si el elemento 9 "Tranquilo... sin prisa, pero sin pausa, seguro lo logro" fuera suprimido, el alfa de Cronbach se acercaría a 0,8 con un valor de 0,7999. En cambio, si el elemento 2 "QQué cansancio! Bueno, tengo que seguir si quiero pasar" fuera suprimido, el alfa de Cronbach bajaría cerca de 0,03. Estas proyecciones son de gran utilidad para verificar la fiabilidad interna del instrumento. Especialmente, porque indican que para el caso de la muestra tomada los reactivos 9 y 2 deberían ser objeto 
de una cuidadosa evaluación que determinará, con ayuda de variados coeficientes, su precisión para medir la autorregulación del aprendizaje.

En el caso de los factores de la autorregulación del aprendizaje, los resultados obtenidos para la medida de coherencia o consistencia interna por cada uno fueron los siguientes: emociones negativas $\alpha 0,718$, motivación $\alpha 0,761$, evitación $\alpha 0,361$, desempeño $\alpha 0,644$ y proceso $\alpha$ 0,641. Las cifras del análisis estadístico descriptivo entre los cinco factores de indagación presentaron las siguientes particularidades: el valor máximo de la media estuvo en la motivación con 3,2100, mientras que la cifra mínima estuvo en la evitación con 1,9375. El rango de la media entre los factores fue de 1,2725.

Para el caso de la desviación estándar, los datos que presentan mayor dispersión en relación con la media son los arrojados del factor de control del proceso. El promedio de dicha desviación es igual a 1,0389 y es la cifra más alta entre los diferentes valores de la desviación presentadas.

\begin{tabular}{|l|c|c|c|c|c|}
\hline & Emociones & Motivación & Evitación & Desempeño & Proceso \\
\hline Media & 2,1938 & 3,2100 & 1,9375 & 3,0167 & 2,8500 \\
\hline Desviación estándar &, 83683 &, 86166 &, 82577 &, 70083 & 1,03898 \\
\hline Mínimo & 1,00 & 1,60 & 1,00 & 1,33 & 1,00 \\
\hline Máximo & 4,00 & 4,80 & 4,50 & 4,17 & 5,00 \\
\hline
\end{tabular}

Tabla 3.

Estadísticos de los factores de la Autorregulación del aprendizaje planteados por Alonso-Tapia y Panadero (2010).

Las cifras de desviación estándar de factores tales como emociones, motivación y evitación son muy similares. Los valores de la dispersión están en $0,83683,0,86166$ y 0,82577, respectivamente. Finalmente, el dato de la desviación estándar más bajo está dentro del factor del control de desempeño con 0,70083 , con una diferencia de 0,33815 en relación con la cifra más alta.

Los coeficientes de correlación de Pearson entre los factores se muestran en la Tabla 4. En cuatro correlaciones el coeficiente (.) fue negativo. En el primer caso, los factores o las variables de motivación y de emociones obtuvieron una cifra de $.=-0,177$. En el segundo caso, el control de procesos y las emociones el coeficiente estuvo sobre -0,032. En el tercer caso, la correlación entre el control de la evitación y la motivación fue de . $=-0,053$ y finalmente, en el último caso, el control de procesos y la evitación muestran una correlación de $-0,131$.

Las correlaciones más significativas entre los factores o variables se muestran con dos colas y fue significativa en el nivel 0,01 en la Tabla 4. En el primer caso, las variables de evitación y emoción presentan una correlación de 0,644. En el segundo caso, las variables de desempeño y emociones alcanzaron un coeficiente de 0,596 , un 0,048 más que el primer caso. En el tercer caso, el coeficiente de correlación de Pearson también es positivo y se muestra en la relación existente entre el control en el proceso y el control de la motivación con un valor . de 0,580. Finalmente, se evidencia la correlación positiva entre el desempeño y la evitación, con 
una cifra de . igual a 0,438. Para el caso de las estadísticas por género presentes en la Tabla 5, se puede identificar que la media más baja está en las mujeres y su relación con la evitación con un valor igual a 1,6667. Esta variable en el caso de los hombres es de 2,2368, una diferencia que sobrepasa los 0,5. Para el caso del desempeño, la situación se invierte muy levemente, pues los hombres obtienen una media de 2,9912 a diferencia de las mujeres que tienen una media 0,0485 mayor que la del género contrario.

\begin{tabular}{|c|c|c|c|c|c|}
\hline & Emociones & Motivación & Evitación & Desempeño & Proceso \\
\hline Emociones & 1 & & & & \\
\hline Motivación &,- 177 & 1 & & & \\
\hline Evitación &, $644^{* *}$ &,- 053 & 1 & & \\
\hline Desempeño &, $596^{* *}$ &, 270 &, $438^{* *}$ & 1 & \\
\hline Proceso &,- 032 &, $580^{* *}$ &,- 131 &, 244 & 1 \\
\hline
\end{tabular}

Tabla 4.

Correlaciones de Pearson entre factores

\begin{tabular}{|c|c|c|c|c|}
\hline & Género & Media & Desviación estándar & Media de error estándar \\
\hline \multirow{2}{*}{ Motivación } & Mujer & 3,2476 &, 82923 &, 18095 \\
\cline { 2 - 5 } & Hombre & 3,1684 &, 91715 &, 21041 \\
\hline \multirow{2}{*}{ Emociones } & Mujer & 2,0595 &, 77824 &, 16983 \\
\cline { 2 - 5 } & Hombre & 2,3421 &, 89447 &, 20521 \\
\hline \multirow{2}{*}{ Evitación } & Mujer & 1,6667 &, 55528 &, 12117 \\
\cline { 2 - 5 } & Hombre & 2,2368 &, 97708 &, 11553 \\
\hline \multirow{2}{*}{ Desempeño } & Mujer & 3,0397 &, 52943 &, 19887 \\
\cline { 2 - 5 } & Hombre & 2,9912 &, 86687 &, 24269 \\
\hline \multirow{2}{*}{ Proceso } & Mujer & 2,9762 & 1,11216 &, 22070 \\
\cline { 2 - 5 } & Hombre & 2,7105 &, 96200 & \\
\hline
\end{tabular}

Tabla 5.

Estadísticas de factores relacionados con el género de los participantes.

Finalmente, se puede observar con las cifras de la Tabla 5 que el comportamiento de las medias es considerablemente similar. A diferencia de las cifras de las medias, con los valores de las desviaciones estándar, se revela que para cada factor de la autorregulación del aprendizaje la dispersión de los datos por género se comporta de forma diferente. Por ejemplo, la desviación estándar de las mujeres en el factor centrado en el proceso es la mayor de toda la medición y está en 1,11216. La diferencia en este mismo factor con la desviación de los hombres es de 0,15016.

El valor mínimo de la desviación estándar se encuentra también en el grupo de las mujeres. Se observa que el factor o variable de desempeño es de 0,52943 , cuya diferencia con el grupo de los hombres es de 0,33744 . Finalmente, se puede concluir que exceptuando el factor de proceso, en todos los casos, los valores de la desviación estándar de las mujeres para los demás factores es menor que el de los hombres.

\section{DISCUSIÓN Y CONCLUSIÓN}

Con la base de los resultados encontrados, en primer lugar, se puede afirmar que la medida de coherencia o consistencia interna 
del Cuestionario de Mensajes Autorregulatorios (CMA) o del SelfRegulatory Messages Questionnaire (SRMQ), para la muestra estudiada, está en el rango de aceptable a bueno, teniendo en cuenta que el coeficiente $\alpha$ supera el 0,7. Esto quiere decir que los ítems del instrumento aplicado están interrelacionados y miden el constructo objeto de indagación.

En segundo lugar, se puede asegurar que las correlaciones más significativas entre los factores o variables de análisis revelan inicialmente cómo la autorregulación centrada en el desempeño varía de acuerdo con el índice de emociones negativas que en determinada tarea o actividad exprese el participante (Rincón-Baez, 2019; Rosário et al., 2014; Zheng et al., 2021). Esto conlleva a reconocer que la baja autorregulación emocional está asociada a altos indicadores de fracaso en tareas y actividades. Al respecto, estudios como el de Rivers, Brackett, Katulak y Salovey (2006), y el de Winne y Hadwin (1998), han demostrado cómo la regulación eficaz de las emociones, especialmente todas aquellas que están asociadas con la negatividad ante el desarrollo de una actividad, está asociada con la mejora en los indicadores de desempeño. En otras palabras, un individuo que autorregule eficazmente su experiencia emocional tendrá mayores posibilidades de conseguir los fines propuestos, ya que al regular la negatividad se facilita la mejora percibida en las metas (Kuhl, 1984; Weiner, 1986).

En ese sentido, se puede plantear que la falta de regulación emocional afecta el desempeño académico, pues menoscaba los procesos de atención, el procesamiento de la información y eleva la ansiedad (Andrade \& Du, 2005; Benbunutti, 2002). Algunos de los efectos de una escasa autorregulación de las emociones tiene que ver con el deterioro sistemático de los procesos cognitivos que requiere un individuo para aprender y desempeñarse adecuadamente en una tarea o actividad (Cóndor-Herrera, 2020). Sobre esto, Weiser y Riggio (2010) y Zimmerman (2000), han revelado que la creencia recurrente de ineficacia académica está reforzada en la falta de autorregulación de las emociones negativas. Esto, desde luego, pone en peligro el desarrollo armónico de la cognición, reduciendo el desempeño e impidiendo el logro.

Dentro de los resultados también fue evidente una correlación significativa entre la emoción y la evitación. Esto, en cierta medida, podría indicar que los participantes que tienen una insuficiente autorregulación de sus emociones experimentan mayoritariamente automensajes centrados en la evitación. Sobre esto, el estudio de Boekaerts y Corno (2005) ha mostrado que el uso de estrategias enfocadas en la evitación suelen ser útiles para reducir el estrés y la ansiedad que genera afrontar tareas o actividades para los que se considera no tener la suficiente destreza (Russell, Baik, Ryan, \& Molloy, 2020). Al respecto, Bandura (1991) y Panadero (2011), han planteado que las actividades que suelen generar efectos negativos en las emociones de los individuos suelen desembocar en evitación. Por ejemplo, si un estudiante cree que "fracasó" en la ejecución y desarrollo de una tarea o actividad, posiblemente sentirá que independientemente del esfuerzo que le imprima nuevamente sus expectativas de éxito serán muy limitadas. 
En ese sentido, la evitación puede llegar a ser sumamente negativa para el aprendizaje. En especial, cuando los "pensamientos negativos hacen que el alumno se bloquee y olvide la consecución de la meta" (Panadero, 2011, p.23). Sobre esto se podría pensar que la evitación es una consecuencia del precario control de la motivación que se tiene por una tarea o actividad. No obstante, esa correlación no fue visible dentro de la muestra, de modo que no se cuenta con datos suficientes para establecer dicho análisis de factores o variables.

Por otro lado, los datos sugieren una correlación entre motivación y control del proceso, que si bien no es significativa sí apunta a considerar que la fuente principal de motivación para autorregularse tiene que ver con la percepción de autoeficacia. Esto está relacionado con la percepción de competencia que un individuo tiene al sentirse idóneo en algo y que, llegado el caso, podría influir en su motivación intrínseca por una tarea (Paris \& Paris, 2001; Zimmerman, Bonner \& Kovach, 1996). En estos términos, se revela que para la muestra estudiada el control de la motivación influye en la eficacia del proceso aprendizaje. Esto significa que un estudiante motivado llega a planear, ejecutar y evaluar las diferentes estrategias que constituyen su proceso de aprendizaje con el fin de alcanzar las metas trazadas en el inicio.

En términos generales, se puede concluir que el instrumento utilizado para medir los mensajes de autorregulación del aprendizaje ayudó a evidenciar las correlaciones existentes entre evitación, emoción, desempeño y control del proceso de aprendizaje. Sin embargo, el reducido número de participantes y el hecho de que el estudio se realizó en una sola institución son dos factores que tienen implicaciones para la validez externa. Otro factor es que solo participó un grupo del grado noveno de la institución (en una sola jornada), por lo que los resultados no se pueden generalizar para toda la institución.

En ese sentido, el estudio no experimental adelantado en el Colegio Cundinamarca IED puede constituirse en un primer acercamiento para indagar a mayor profundidad qué opiniones y actitudes surgen a manera de auto-mensajes en la mente de los estudiantes mientras desarrollan algún tipo de tarea o actividad. Esto podría ser de gran ayuda para delinear las características clave de los procesos de autorregulación del aprendizaje que en la actualidad están desarrollando los estudiantes de grado noveno en la institución.

Finalmente, se debe subrayar que para la muestra estudiada, las correlaciones más significativas estuvieron vinculadas con la emoción. Esto podría sugerir que el control de las emociones está asociado congruentemente con el uso de estrategias de evitación y desempeño. Se espera que para próximos estudios se logre ahondar con mayor profundidad las correlaciones significativas que este tipo de factores pueden tener en la mejora de la autorregulación. 


\section{REFERENCIAS}

Ader, E. (2019). What would you demand beyond mathematics? Teacher's' promotion of students' self-regulated learning and metacognition. ZDM Mathematics Education, 51, 613-624. https://doi.org/10.1007/s11858-0 19-01054-8

Aguilar, V. (2020). Autorregulación docente. Modelos para el fortalecimiento e investigación de la docencia. Octaedro.

Alonso-Tapia, J., \& Panadero, E. (2010). Effect of self-assessment script on self-regulation and learning. Journal for the Study of Education and Development, 33(3), 385-397. https://doi.org/10.1174/0210370107922 15145

Andrade, H. \& Du, Y. (2005). Student perspectives on rubric-referenced assessment. Practical Assessment, Research \& Evaluation, 10(3), 1-13. htt ps://doi.org/10.7275/g367-ye94

Bandura, A. (1991). Social cognitive theory of self-regulation. Organizational Behaviour and Human Decision Processes, 50(2), 248-287. https://doi.org /10.1016/0749-5978(91)90022-L

Battaglia, M. (2008). Response rates: how have the changed and where are they headed? In J.M. Lepkowski et.al. (Eds.), Advances in telephone survey methodology. (pp.529-560). Wiley.

Benbunutti, H. (2002). Emotion regulation and test anxiety: the contribution of academic delay of gratification. Annual Meeting of the American Educational Research Association. Paper presentation.

Boekaerts, M. \& Corno, L. (2005). Self-regulation in the classroom: A perspective on assessment and intervention. Applied Psychology an International Review, 54(2), 199-231. https://doi.org/10.1111/j.1464-0 597.2005.00205.x

Boekaerts, M., Pintrich, P. \& Zeidner, M. (Eds.) (2000). Handbook of selfregulation. Academic Press.

Cabero-Almenara, J. (2020). Aprendiendo del tiempo de la COVID-19. Revista Electrónica Educare, 24(1). Recuperado de: https://www.redalyc.org/jou $\mathrm{rnal} / 1941 / 194165541002 / 194165541002 . \mathrm{pdf}$

Cóndor-Herrera, O. (2020). Educar en tiempos de COVID-19. CienciAmérica, 9(2), 31. https://doi.org/10.33210/ca.v9i2.281

Corno, L. (2008). Work habits and self-regulated learning: Helping students to find a "Will" from a "Way". In: D, Schunk \& B. Zimmerman. (Eds.). Motivation and self-regulated learning. Theory, research and applications (pp.197-222). Lawrence Erlbaum Associates.

De Corte, E., Verschaffel, L., \& Eynde, P. (2000). Self-regulation: A characteristic and a goal of mathematics education. In M. Boekaerts, P.R. Pintrich, \& M. Zeidner (Eds.). Handbook of self-regulation (pp. 687-726). Academic Press.

Dignath, C., \& Büttner, G. (2008). Components of fostering self-regulated learning among students. A meta-analysis on intervention studies at primary and secondary school level. Metacognition and Learning, 3(3), 231-264. https://doi.org/10.1007/s11409-008-9029-x

Föster, C. (2018). El poder de la evaluación en el aula: mejores decisiones para promover aprendizajes. Ediciones UC. 
Hernández, R., Fernández, C., \& Baptista, M. (2014). Metodología de la investigación. McGraw-Hill.

Kuhl, J. (1984). Volitional aspects of achievement motivation and learned helplessness: Toward a comprehensive theory of action-control. In: A. Maher. (Ed.). Progress in experimental personality research (pp. 99-171). Academic Press.

Kuhl,J. (2000). A functional-design approach to motivation and self-regulation. In: M. Boekaerts, P. Pintrich, \& M. Zeidner (Eds.). Handbook of selfregulation (pp.111-169). Academic Press.

Mega, C., Ronconi, L., \& De Beni, R. (2014). What makes a good student? How emotions, self-regulated learning, and motivation contribute to academic achievement. Journal of Educational Psychology, 106(1), 121-131. https:/ /doi.org/10.1037/a0033546

Norman-Acevedo, E., Daza-Orozco , CE., \& Caro-Gómez, CL. . (2021). Hoja de ruta para la elaboración de resultados de aprendizaje para la formación investigativa. Panorama, 15(28), 1-12. https://doi.org/10.15765/pnrm. v15i28.1813

Norman-Acevedo, E., \& Daza-Orozco, CE. (2020). La construcción de contenidos para la enseñanza virtual: retos coyunturales en el confinamiento. Panorama, 14(27), 5-13. https://doi.org/10.15765/pnr m.v14i 27.1517

Ortega, M. (2020). Autorregulación de las interacciones escritoras: una aproximación metodológica con universitarios. Revista Brasileira de Educação, 25. Recuperado de: https://www.redalyc.org/journal/275/275 63097039/27563097039.pdf

Panadero, E. (2011). Ayudas instruccionales a la autoevaluación y la autorregulación: evaluación de la eficacia de los guiones de autoevaluación frente a la de las rúbricas. Universidad Autónoma de Madrid.

Paris, S., \& Paris, A. (2001). Classroom applications of research on self-regulated learning. Educational Psychologist, 36(2), 89-101. https://doi.org/10.120 7/S15326985EP3602_4

Pintrich, P. (2000). An achievement goal theory perspective on issues in motivation terminology, theory, and research. Contemporary Educational Psychology, 25, 92-104. https://doi.org/10.1006/ceps.1999.1017

Rincón-Baez, W. (2019). Brecha de rendimiento académico por género en Saber Pro en programas de administración en los departamentos de Colombia. Revista Panorama, 13(25). Recuperado de: https://www.redalyc.org/jou $\mathrm{rnal} / 3439 / 343963314012 / 343963314012 . \mathrm{pdf}$

Ríos, D. (2020). La descentralización de la práctica evaluativa orientada al autoaprendizaje del estudiante. Educação e Pesquisa, 46. Recuperado de: h ttps://www.redalyc.org/journal/298/29863344002/29863344002.pdf

Rivers, S., Brackett, M., Katulak, N., \& Solovey, P. (2007). Regulating anger and sadness: an exploration of discrete emotions in emotion regulation. Journal of Happiness Studies, 8, 397-427. https://doi.org/10.1007/s1090 2-006-9017-2

Rosário, P., Pereira, A., Högemann, J., Nunes, A., Figueiredo, M., Núñez, J., ... Gaeta, M. (2014). Autorregulación del aprendizaje: Una revisión sistemática en revistas de la base SciELO. Universitas Psychologica, 13(2), 781-798. https://doi.org/10.11144/Javeriana.UPSY13-2.aars 
Ruipérez-Valiente, J. (2020). El Proceso de Implementación de Analíticas de Aprendizaje. RIED. Revista Iberoamericana de Educación a Distancia, 23(2). Recuperado de: https://www.redalyc.org/journal/3314/3314631 71005/331463171005.pdf

Ruban, L. \& Reis, S. (2006). Patterns of self-regulation: Patterns of Self-regulatory Strategy Use among Low-Achieving and HighAchieving University Students. Roeper Review, 28, 148-156. DOI: 10.1080/02783190609554354

Russell, J., Baik, C., Ryan, A., \& Molloy, E. (2020). Fostering self-regulated learning in higher education: Making self-regulation visible. Active Learning in Higher Education. https://doi.org/10.1177/1469787420982 378

Schunk, D. (2001). Social cognitive theory and self-regulated learning. In: B, Zimmerman \& D. Schunk (Eds.). Self-regulated learning and academic achievement (pp.125-151). Lawrence Erlbaum Associates.

Schunk, D. (2012). Teorias del aprendizaje. Una perspectiva educativa. Pearson.

Spruce, R., \& Bol, L. (2015). Teacher beliefs, knowledge, and practice of selfregulated learning. Metacognition and Learning, 10(2), 245-277. https:// doi.org/10.1007/s11409-014-9124-0

Weiner, B. (1986). An attributional theory of motivation and emotion. SpringerVerlag.

Weiser, D., \& Riggio, H. (2010). Family background and academic achievement: does self-efficacy mediate outcomes? Social Psychology of Education, 13(3), 367-383. https://doi.org/10.1007/s11218-010-9115-1

Winne, P. \& Hadwin, A. (1998). Studying as self-regulated learning. In: D. Hacker, J. Dunlosky \& A. Graesser (Eds.). Metacognition in educational theory and practice (pp. 277-304). Erlbaum.

Wolters, C. (2003). Regulation of motivation: evaluating an underemphasized aspect of self-regulated learning. Educational Psychologist, 38(4), 189-205. https://doi.org/10.1207/S15326985EP3804_1

Wolters, C. (2003). Regulation of motivation: Evaluating an underemphasized aspect of self-regulated learning. Educational Psychologist, 38(4), 184-205. https://doi.org/10.1207/S15326985EP3804_1

Zimmerman, B. (2000). Attaining self-regulation: a social cognitive perspective. In: M. Boekaerts, P. Pintrich \& M. Zeidner (Eds.). Handbook of selfregulation (pp. 13-39). Academic Press.

Zimmerman, B. (2001). Theories of self-regulated learning and academic achievement: an overview and analysis. In B.J. Zimmerman \& D.H. Schunk (Eds.). Self-regulated learning and academic achievement (pp.1-37). Lawrence Erlbaum Associates.

Zimmerman, B. (2002). Becoming a self-regulated learner: An Overview. Theory into Practice, 41, 64-70. https://doi.org/10.1207/s15430421 tip4102_2

Zimmerman, B. (2008). Goal setting: a key proactive source of academic selfregulation. In D.H. Schunk \& B.J. Zimmerman (Eds.). Motivation and self-regulated learning. Theory, research and applications (pp.267-295). Lowrence Erlbaum Associates.

Zimmerman, B., Bonner, S., \& Kovach, R. (1996). Developing self-regulated learners: Beyond achievement to self-efficacy. American Psychological Association. 
Zheng, J., Huang, L., Li, S., Lajoie, S., Chen, Y., \& Hmelo-Silver, C. (2021). Selfregulation and emotion matter: A case study of instructor interactions with a learning analytics dashboard. Computers and Education, 161. http s://doi.org/10.1016/j.compedu.2020.104061 